\title{
An analogue of the Thue-Morse sequence
}

\author{
Emmanuel FERRAND \\ Institut Mathématique de Jussieu UMR CNRS 7586, \\ Université Pierre et Marie Curie Paris VI, \\ Case 247 - 4, place Jussieu - 75252 Paris Cedex, \\ FRANCE \\ ferrand@math.jussieu.fr
}

Submitted: Sep 21, 2005; Accepted: Feb 23, 2007; Published: Apr 23, 2007

Mathematics Subject Classification: 05C 88

\begin{abstract}
We consider the finite binary words $Z(n), n \in \mathbb{N}$, defined by the following selfsimilar process: $Z(0):=0, Z(1):=01$, and $Z(n+1):=Z(n) \cdot \overline{Z(n-1)}$, where the dot $\cdot$ denotes word concatenation, and $\bar{w}$ the word obtained from $w$ by exchanging the zeros and the ones. Denote by $Z(\infty)=01110100 \ldots$ the limiting word of this process, and by $z(n)$ the $n$ 'th bit of this word. This sequence $z$ is an analogue of the Thue-Morse sequence. We show that a theorem of Bacher and Chapman relating the latter to a "Sierpiński matrix" has a natural analogue involving $z$. The semi-infinite self-similar matrix which plays the role of the Sierpiński matrix here is the zeta matrix of the poset of finite subsets of $\mathbb{N}$ without two consecutive elements, ordered by inclusion. We observe that this zeta matrix is nothing but the exponential of the incidence matrix of the Hasse diagram of this poset. We prove that the corresponding Möbius matrix has a simple expression in terms of the zeta matrix and the sequence $z$.
\end{abstract}

\section{Introduction}

Consider the finite binary words $T(n), n \in \mathbb{N}$, defined by the following self-similar process: $T(0):=0$, and $T(n+1):=T(n) \cdot \overline{T(n)}$, where the dot $\cdot$ denotes word concatenation, and $\bar{w}$ the word obtained from $w$ by exchanging the zeros and the ones. Denote by $T(\infty)=01101001 \ldots$ the limiting word of this process, and by $t(n)$ the $n$ 'th bit of this word. The sequence $t$ is often called the Thue-Morse sequence and has appeared in various fields of mathematics. See, for example, the paper [AS1], which contains a review of the main properties of this sequence and which is a good starting point to the abundant literature on the subject ${ }^{1}$.

\footnotetext{
${ }^{1}$ See also $[\mathrm{AS} 2,6.2]$
} 
In $[\mathrm{BC}]$, Bacher and Chapman showed how the Thue-Morse sequence appears in the context of $L D U$ decomposition of self-similar matrices. Their result [BC, Theorem 1.1] can be rephrased as follows: Denote by $S$ the symmetric semi-infinite matrix whose entries are in $\{0,1\}$ and such that $S_{i, j} \equiv\left(\begin{array}{c}i+j \\ j\end{array}\right)(\bmod 2),(i, j) \in \mathbb{N}^{2}$. Denote by $B$ the semi-infinite lower triangular matrix whose entries are in $\{0,1\}$ and such that $B_{i, j} \equiv\left(\begin{array}{l}i \\ j\end{array}\right)$ $(\bmod 2),(i, j) \in \mathbb{N}^{2}$.

$$
B=\left(\begin{array}{ccccc}
1 & 0 & 0 & 0 & \ldots \\
1 & 1 & 0 & 0 & \ldots \\
1 & 0 & 1 & 0 & \ldots \\
1 & 1 & 1 & 1 & \ldots \\
\vdots & \vdots & \vdots & \vdots & \ddots
\end{array}\right) \quad S=\left(\begin{array}{ccccc}
1 & 1 & 1 & 1 & \ldots \\
1 & 0 & 1 & 0 & \ldots \\
1 & 1 & 0 & 0 & \ldots \\
1 & 0 & 0 & 0 & \ldots \\
\vdots & \vdots & \vdots & \vdots & \ddots
\end{array}\right)
$$

Due to their self-similar properties (see below), both $B$ and $S$ can be considered as matrix versions of the Sierpiński sieve ${ }^{2}[\mathrm{Si}$ ], and $B$ deserves the name Sierpiński matrix.

Denote by $D$ the semi-infinite diagonal matrix whose non-zero entries are $D_{i, i}=$ $(-1)^{t(i)}, i \in \mathbb{N}$. According to Bacher and Chapman [BC, Theorem 1.1 and Theorem 2.1],

$$
S=B D B^{T}
$$

In this note we are interested in the following mixture of the Thue-Morse sequence and the Fibonacci word ${ }^{3}$, introduced by Shallit [Sh, Example 2, p. 12]: Consider the finite binary words $Z(n), n \in \mathbb{N}$, defined by the following self-similar process: $Z(0):=0$, $Z(1):=01$, and $Z(n+1):=Z(n) \cdot \overline{Z(n-1)}$. Denote by

$$
Z(\infty)=0111010010001100010111000101101110 \ldots
$$

the limiting word of this process ${ }^{4}$, and by $z(n)$ the $n$ 'th bit of this word (so that $z(0)=0$, $z(1)=1$, etc...).

We will show that a natural analogue of equation (1) involves our sequence $z$. For this we will introduce two semi-infinite self-similar matrices, which will play the role of $S$ and $B$ above. The Thue-Morse sequence and the matrices $S$ and $B$ can be generalized in many different natural ways. The main point of this note lies in the choice of the definitions of our analogues for $S$ and $B$. This choice will be inspired by the theory of partially ordered sets. Thanks to these "good" definitions, the proofs will be straightforward. Another result of this note is the observation that the matrix $B$ and its analogue have remarkable logarithms and inverses (see section 3).

\footnotetext{
${ }^{2} \mathrm{~A}$ classical example of a fractal set, also called the Sierpiński gasket [Ma], not to be taken for its cousin the Sierpiński carpet [AS2, 14.1].

${ }^{3}$ See, for example, [AS2, 7.1] for an introduction to the Fibonacci word.

${ }^{4}$ The referee observed that $Z(\infty)$ can be written as the fixed point of the morphism $0 \rightarrow 01,1 \rightarrow 3,3 \rightarrow$ $32,2 \rightarrow 0$, followed by taking the result mod 2 . This can be proved using the fact that this morphism commutes with the involution $0 \rightarrow 3,1 \rightarrow 2,2 \rightarrow 1,3 \rightarrow 0$.
} 

$[\mathrm{AS} 1])$ :

We start by the following interpretation of the Thue-Morse sequence (see, for example,

Denote by $|n|$ the number of 1's in the binary expansion of $n$.

Lemma 1. $t(n) \equiv|n|(\bmod 2)$

With a finite subset $K \subset \mathbb{N}$, associate the integer $n(K)=\sum_{k \in K} 2^{k}$.

Lemma 2. $B_{n(K), n(J)}=1$ if and only if $J \subset K$.

Proof. A theorem of Lucas (see, for example, [GKP, ex. 61, p. 248]), permits us to determine the parity of $\left(\begin{array}{c}n \\ m\end{array}\right)$ in terms of the binary expansion of $n$ and $m$ as follows: write $n=\sum_{i=0}^{i=N} \epsilon_{i} 2^{i}, m=\sum_{i=0}^{i=N} \eta_{i} 2^{i}$. Then we have

$$
\left(\begin{array}{c}
n \\
m
\end{array}\right) \equiv \prod_{i=0}^{i=N}\left(\begin{array}{c}
\epsilon_{i} \\
\eta_{i}
\end{array}\right) \quad(\bmod 2)
$$

For each $i, \epsilon_{i}$ and $\eta_{i}$ are either 0 or 1 . Hence $\left(\begin{array}{c}\epsilon_{i} \\ \eta_{i}\end{array}\right)=1$ if and only if $\epsilon_{i} \eta_{i}=\eta_{i}$. In other words, if $n=\sum_{k \in K} 2^{k}$ and $m=\sum_{j \in J} 2^{j}$, then $\left(\begin{array}{c}n \\ m\end{array}\right) \equiv 1(\bmod 2)$ if and only if $J \subset K$.

Remark 1. It is easy to see that $B B^{T} \equiv S(\bmod 2)$ (by Vandermonde convolution, [GKP, p. 174]). Hence the result of Bacher and Chapman (equation (1) above) just explains what correction should be inserted between $B$ and $B^{T}$ to turn the above congruence into an equality valid over the integers.

The length of the word $Z(n)$ is, by construction, the $(n+2)$ 'th Fibonacci number $F(n)$, assuming the usual convention $F(1)=F(2)=1$. Hence it is not unexpected that the expansion of natural numbers described below will play here a role similar to the one played by the binary expansion in the preceding discussion:

Lemma 3. [Ze][AS2, 3.8] Any natural number is uniquely represented as a sum of nonconsecutive Fibonacci numbers of index larger than 1.

Definition 1. The Zeckendorf expansion of $n$ is the unique finite subset $\zeta_{n}$ of $\mathbb{N}$ without two consecutive elements such that $n=\sum_{k \in \zeta_{n}} F(k+2)$.

Denote by $|\mathcal{S}|$ the cardinality of a finite set $\mathcal{S}$.

Lemma 4. $z(n) \equiv\left|\zeta_{n}\right|(\bmod 2)$.

Proof. Given some $n \in \mathbb{N}$, denote by $l$ the largest element in $\zeta_{n}: F(2+l)$ is the largest Fibonacci number not larger than $n$. This implies that $n=m+F(2+l)$ with $m<F(1+l)$. Otherwise, $n$ would be of the form $m_{0}+F(1+l)+F(2+l)=m_{0}+F(3+l)$. This would contradict the fact that $F(2+l)$ is the largest Fibonacci number not larger than $n$. It follows from the definition of $z$ that $z(n)=z(m+F(2+l))=\overline{z(m)}$. On the other hand $\left|\zeta_{n}\right|=\left|\zeta_{m}\right|+1$. The lemma follows by induction. 


\section{Some self-similar matrices.}

The theory of partially ordered sets (posets in the remaining of this paper) will guide us to produce an analogue of equation (1) involving our sequence $z$. Denote by $\mathcal{B}$ the Boolean poset, whose elements are the finite subsets of $\mathbb{N}$, ordered by inclusion. For all $k \in \mathbb{N}$, denote by $\mathcal{B}_{k}$ the poset whose elements are the subsets of $\{0, \ldots, k-1\}$, ordered by inclusion.

A relationship between posets and matrices is provided by the following definition.

Definition 2. The zeta matrix ${ }^{5}$ of a countable poset $\mathcal{P}$ is the matrix whose rows and columns are indexed by the elements of $\mathcal{P}$, with an entry 1 in row $x$ and column $y$ if $x \leq y$, and 0 otherwise.

Example 1. Lemma 2 above can be rephrased as follows. $B$ is the zeta matrix of $\mathcal{B}$ (up to the identification of an element $K \in \mathcal{B}$ by $n(K) \in \mathbb{N}$ ).

Let us introduce a self-similar matrix $A$, which appears to be natural in our context. Consider the poset $\mathcal{A}$ whose elements are those finite subsets of $\mathbb{N}$ without two consecutive elements, ordered by inclusion. Denote by $\mathcal{A}_{k}$ the poset $\mathcal{A} \cap \mathcal{B}_{k}$. Notice that the Zeckendorf expansion realizes a one-to-one correspondence between the elements of $\mathcal{A}$ and the natural numbers, which permits us to identify the elements of $\mathcal{A}$ with the integers. Denote by $A$ the zeta matrix of $\mathcal{A}: A_{i, j}=1$ iff $\zeta_{j} \subset \zeta_{i}$, and 0 otherwise.

To understand in what sense the matrix $A$ is self-similar, let us introduce the following sequence of matrices $A(k), k \in \mathbb{N}$.

$$
A(0)=(1), A(1)=\left(\begin{array}{ll}
1 & 0 \\
1 & 1
\end{array}\right) \text {, and, given an integer } k \text {, define } A(k+1) \text { to be the square, }
$$

lower triangular matrix of size $F(k+3)$, recursively defined by

$$
A(k+1)=\left(\begin{array}{ccc}
A(k) & \mathbf{0}_{F(k+2), F(k+1)} \\
A(k-1) & \mathbf{0}_{F(k+1), F(k)} & A(k-1)
\end{array}\right)
$$

where $\mathbf{0}_{p, q}$ denotes a rectangular block of zeros with $p$ rows and $q$ columns.

$$
\text { For example } A(2)=\left(\begin{array}{lll}
1 & 0 & 0 \\
1 & 1 & 0 \\
1 & 0 & 1
\end{array}\right) \text {, and } A(3)=\left(\begin{array}{lllll}
1 & 0 & 0 & 0 & 0 \\
1 & 1 & 0 & 0 & 0 \\
1 & 0 & 1 & 0 & 0 \\
1 & 0 & 0 & 1 & 0 \\
1 & 1 & 0 & 1 & 1
\end{array}\right) \text {. }
$$

\footnotetext{
${ }^{5}$ The inverse of this matrix is classically called the "Möbius matrix" of $\mathcal{P}$. See for example [Bó]. The classical Möbius inversion formula can be interpreted in terms of the Möbius matrix corresponding to the divisibility order of the integers . The relationship between the Möbius inversion formula and Riemann's zeta function motivates the use of the name "zeta matrix" in the context of a general poset.
} 
Lemma 5. For all $k \in \mathbb{N}, A(k)$ is the zeta matrix of $\mathcal{A}_{k}$ (up to the identification of $\mathcal{A}_{k}$ with $\{0, \ldots, F(k+2)-1\} \subset \mathbb{N}$ via the Zeckendorf expension).

Proof. This is true for $k=1$. Assume that the claim is true for some $k$, and let us show that $A(k+1)_{s, t}=1$ iff $\zeta_{t} \subset \zeta_{s}$. If $s<F(k+2)$, then a non-zero entry $A(k+1)_{s, t}$ implies that $t \leq s$. Hence $A(k+1)_{s, t}$ can be interpreted as an entry of $A(k)$, and the statement holds by the induction hypothesis. Suppose now that $F(k+2) \leq s<F(k+3)$. We have that $s^{\prime}=s-F(k+2)<F(k+3)-F(k+2)=F(k+1)$. In other words, $\zeta_{s}=\zeta_{s^{\prime}} \cup\{k\}$. Hence $\zeta_{t} \subset \zeta_{s}$ if and only if $\zeta_{t} \subset \zeta_{s^{\prime}}$ or $\zeta_{t} \subset \zeta_{s^{\prime}} \cup\{k\}$. The first case is reflected in the left lower block of $A(k+1)$. The last case corresponds to the diagonal lower block of $A(k+1)$.

Now let us introduce another sequence of matrices $R(k), k \in \mathbb{N}$, with entries in $\mathbb{Z}[X]$.

$R(0)=(1), R(1)=\left(\begin{array}{ll}1 & 1 \\ 1 & X\end{array}\right)$, and, given an integer $k, R(k+1)$ is the square symmetric matrix of size $F(k+3)$, recursively defined by

$$
R(k+1)=\left(\begin{array}{lc}
R(k) & \underline{R}(k)^{T} \\
\underline{R}(k) & X \cdot R(k-1)
\end{array}\right),
$$

where $\underline{R}(k)$ stands for the $F(k+1) \times F(k+2)$ matrix obtained by removing the last $F(k)$ rows of $R(k)$.

For example, $R(2)=\left(\begin{array}{ccc}1 & 1 & 1 \\ 1 & X & 1 \\ 1 & 1 & X\end{array}\right)$, and $R(3)=\left(\begin{array}{ccccc}1 & 1 & 1 & 1 & 1 \\ 1 & X & 1 & 1 & X \\ 1 & 1 & X & 1 & 1 \\ 1 & 1 & 1 & X & X \\ 1 & X & 1 & X & X^{2}\end{array}\right)$.

For all $k \in \mathbb{N}$, denote by $C(k)$ the $F(k+2) \times F(k+2)$ diagonal matrix with entries in $\mathbb{Z}[X]$, whose non-zero entries are $C(k)_{l, l}=(X-1)^{\left|\zeta_{l}\right|}, l \in\{0, \ldots, F(k+2)-1\}$.

Theorem 1. For any $k \in \mathbb{N}, R(k)=A(k) C(k) A(k)^{T}$.

Proof. This is true for $k=1$. Assume that this is true up to some integer $k$. Observe that

$$
C(k+1)=\left(\begin{array}{cc}
C(k) & 0 \\
0 & (X-1) \cdot C(k-1)
\end{array}\right) .
$$

A block-wise computation of the product $A(k+1) C(k+1) A(k+1)^{T}$ shows that it satisfies the recurrence relation that defines $R(k+1)$. 
An application. Denote by $\Sigma$ the symmetric (semi-infinite) matrix with coefficients in $\{0,1\}$ such that $\Sigma \equiv A A^{T}(\bmod 2)$. Induction shows the following result.

Lemma 6. $\Sigma$ is the limiting matrix of the family $R(k), k \in \mathbb{N}$, when the variable $X$ is evaluated at 0 .

Theorem 1 then implies that

$$
\Sigma=A C A^{T}
$$

where $C$ is the diagonal matrix whose $i$ 'th diagonal entry is $C_{i, i}=(-1)^{z(i)}, i \in \mathbb{N}$. This is the analogue of the $L D U$ decomposition of Bacher and Chapman (equation (1)) discussed earlier.

A "Boolean" version. For completeness, we mention a "Boolean" version of Theorem 1 , which is implicit in $[\mathrm{BC}]$. It deals with the following families $B(k)$ and $S(k), k \in \mathbb{N}$ of matrices of size $2^{k}$, respectively lower triangular and symmetric. The matrices $B(0)=$ $S(0)$ are both equal to the 1 by 1 identity matrix, and $B(k+1)$ and $S(k+1)$ are recursively defined by:

$$
B(k+1)=\left(\begin{array}{cc}
B(k) & 0 \\
B(k) & B(k)
\end{array}\right), \text { and } S(k+1)=\left(\begin{array}{cc}
S(k) & S(k) \\
S(k) & X \cdot S(k)
\end{array}\right) .
$$

In other words, $B(k)=B(1)^{\otimes k}$ (the $k$ 'th power of $B(1)$ with respect to the Kronecker product of matrices, see [HJ, 4.2]), and $S(k)=S(1)^{\otimes k}$. Of course, the matrix $B(k)$ is the zeta matrix of $\mathcal{B}_{k}: B(k)_{\sum_{i \in I} 2^{i}, \sum_{j \in J} 2^{j}}=1$ iff $J \subset I \subset\{0, \ldots, k-1\}$. For all $k \in \mathbb{N}$, denote by $D(k)$ the $2^{k} \times 2^{k}$ diagonal matrix whose non-zero entries are $D_{l, l}(k)=(X-1)^{|l|}$, $l \in\left\{0 \ldots 2^{k}-1\right\}$. These matrices are "auto-similar" in the sense of Bacher and Chapman, and their result $[\mathrm{BC}$, theorem 2.1] applied to this particular case yields the following:

Theorem 2. For any $k \in \mathbb{N}, S(k)=B(k) D(k) B(k)^{T}$.

Since $t(l) \equiv|l|(\bmod 2)$, the case $X=0$ corresponds to equation (1). Observe also that when the variable $X$ is evaluated at $-1, S(k)$ becomes

$$
B(k)\left(\begin{array}{cc}
1 & 0 \\
0 & -2
\end{array}\right)^{\otimes k} B(k)^{T} .
$$

This is a Hadamard matrix, that is an orthogonal matrix with coefficients in $\{-1,1\}$, introduced and studied in 1867 by Sylvester [Sy]. 


\section{Inverses and logarithms of these zeta matrices.}

Denote by $I$ the semi-infinite identity matrix. Recall that the formula

$$
\ln (I+M)=\sum_{l=1}^{\infty} \frac{(-1)^{l+1}}{l} M^{l}
$$

makes sense for any strictly lower triangular semi-infinite matrix $M$, since the computation of a given element of $\ln (I+M)$ involves only a finite sum. We will call $m=\ln (I+M)$ the logarithm of $I+M$. It is itself a strictly lower triangular semi-infinite matrix. The classical formula $e^{m}=\sum_{l=0}^{\infty} \frac{m^{l}}{l !}$ makes sense for such a semi-infinite matrix, and $e^{m}=I+M$.

In particular it makes sense to consider the logarithms of the zeta matrices considered so far. We will see that these logarithms enjoy a notable property (theorem 3 below) related to the poset structure.

Consider two elements $x$ and $y$ of some poset. One says that $x$ covers $y$ if $y<x$ and if there is no $z$ such that $y<z<x$. A maximal $k$-chain in a poset is a subset $\left\{x_{0}, x_{1}, \ldots, x_{k}\right\}$ such that $x_{p}$ covers $x_{p+1}$ for all $0 \leq p<k$. By the Hasse matrix of a poset, we mean the incidence matrix of its Hasse diagram, viewed as a directed graph: The rows and the columns of this matrix are indexed by the elements of the poset, and the matrix element indexed by a pair $(x, y)$ is 1 if $x$ covers $y$, and 0 otherwise.

Theorem 3. For any $k \in \mathbb{N}, B(k)$ and $A(k)$ have a logarithm with entries in $\{0,1\}$. More precisely, $B=e^{H}$ and $A=e^{G}$, where $H$ and $G$ are the Hasse matrices of the posets $\mathcal{B}$ and $\mathcal{A}$.

Theorem 3 is a corollary of Lemma 7 below, in view of which we introduce the following terminology.

Definition 3. An injective mapping $\phi: \mathcal{Q} \rightarrow \mathcal{R}$ between two posets $\mathcal{Q}$ and $\mathcal{R}$ is called an ideal embedding if for any $x \in \mathcal{Q}$,

$$
\phi(\{y \in \mathcal{Q} \text { such that } y \leq x\})=\{z \in \mathcal{R} \text { such that } z \leq \phi(x)\} .
$$

Example 2. The natural inclusion $\mathcal{A} \subset \mathcal{B}$ is an ideal embedding.

Lemma 7. If a poset $\mathcal{Q}$ can be ideally embedded in the Boolean poset $\mathcal{B}$, then its zeta matrix is the exponential of its Hasse matrix.

Proof. We will use below the letters $G$ and $A$ to denote the Hasse matrix and the zeta matrix of $\mathcal{Q}$. For any $k \in \mathbb{N}$, the entry $G_{i, j}^{k}$ is equal to the number of maximal $k$-chains $\left\{x_{0}, x_{1}, \ldots, x_{k}\right\}$ such that $x_{0}=i, x_{k}=j$. The key point is that if $\mathcal{Q}$ has an ideal embedding in $\mathcal{B}$, then any interval $[i, j]$ in $\mathcal{Q}$ is isomorphic (as a poset) to $\mathcal{B}_{l}$, where $l=|\phi(i)|-|\phi(j)|$. 
In $\mathcal{B}_{l}$, there are exactly $l$ ! maximal $l$-chains from the full set $\{0, \ldots, l-1\}$ down to the empty set. Hence $G_{i, j}^{k}=l$ ! if $k=l$, and vanishes otherwise. In other words, for all $k \in \mathbb{N}$, the matrices $\frac{1}{k !} G^{k}$ have entries in $\{0,1\}$ and have disjoint support.

On the other hand, the entry $A_{i, j}$ is by definition equal to 1 if and only if there exists a maximal $k$-chain from $i$ to $j$, for some $k \in \mathbb{N}$. In other words, $A_{i, j}=1$ if and only if there exists $k$ such that $G_{i, j}^{k}$ is non-zero. This proves that $A=\sum_{k \in \mathbb{N}} \frac{1}{k !} G^{k}$.

An explicit formula. It is interesting to give an alternate (longer) proof of Lemma 7, which has the advantage of providing the explicit formulas of $H$ and $G$. There are two steps. The first one consists in proving that $B=e^{H}$.

In addition to the basic properties of the Kronecker product of matrices (see [HJ, 4.2]) we will need the following key identity [HJ, 4.2.10]: $(A \otimes B)(C \otimes D)=(A C) \otimes(B D)$, where $A, B, C, D$ are four rectangular matrices such that the matrix products involved in this identity make sense. Recall that the zeta matrix of $\mathcal{B}_{k}$ is $B(k)=B(1)^{\otimes k}$. Notice that $B(1)=e^{H(1)}$, where $H(1)=\left(\begin{array}{ll}0 & 0 \\ 1 & 0\end{array}\right)$ is the Hasse matrix of $\mathcal{B}_{1}$, the poset with two comparable elements. Now

$$
B(2)=B(1) \otimes B(1)=e^{H(1)} \otimes e^{H(1)}=\left(e^{H(1)} \otimes I(1)\right) \cdot\left(I(1) \otimes e^{H(1)}\right),
$$

where $I(m)$ denotes the $2^{m} \times 2^{m}$ identity matrix. But $e^{H(1)} \otimes I(1)=e^{H(1) \otimes I(1)}$, and similarly $I(1) \otimes e^{H(1)}=e^{I(1) \otimes H(1)}$. Hence $L(2)=e^{H(1) \otimes I(1)} \cdot e^{I(1) \otimes H(1)}$. The two matrices $H(1) \otimes I(1)$ and $I(1) \otimes H(1)$ commute, so that $B(2)=e^{H(1) \otimes I(1)+I(1) \otimes H(1)}$. This suggests defining, for any $l>0$, the $2^{l+1} \times 2^{l+1}$ matrix $H(l+1)$ by

$$
H(l+1)=I(1) \otimes H(l)+H(1) \otimes I(l)=\left(\begin{array}{cc}
H(l) & 0 \\
I(l) & H(l)
\end{array}\right) .
$$

One can check that, for all $k \in \mathbb{N}, H(k)=\sum_{l=0}^{k-1} I(l) \otimes H(1) \otimes I(k-1-l)$.

On one hand, the recursion above is precisely the one that describes the relationship between the Hasse matrices of the posets $\mathcal{B}_{l}$ and $\mathcal{B}_{l+1}$, and $H(l)$ is indeed the Hasse matrix of $\mathcal{B}_{l}$.

On the other hand, the matrices $I(1) \otimes H(l)$ and $H(1) \otimes I(l)$ commute, so that

$$
\begin{gathered}
e^{H(l+1)}=e^{I(1) \otimes H(l)} \cdot e^{H(1) \otimes I(l)}=\left(I(1) \otimes e^{H(l)}\right)\left(e^{H(1)} \otimes I(l)\right)= \\
=e^{H(1)} \otimes e^{H(l)}=B(1) \otimes e^{H(l)} .
\end{gathered}
$$

Since $B(l+1)=B(1) \otimes B(l)$, we get that $e^{H(l)}=B(l)$ by induction. From this we recover the fact that $B=e^{H}$.

Now consider an ideal embedding $\phi$ of $\mathcal{Q}$ in $\mathcal{B}$. Denote by $U$ the matrix whose rows (resp. columns) are indexed by the elements of $\mathcal{Q}($ resp. $\mathcal{B})$ and whose entries are $U_{i, j}=1$ 
if $j=\phi(i)$, and 0 otherwise. Since the order on $\mathcal{Q}$ is induced by $\Phi$ from the order on $\mathcal{B}$, we have that

$$
A=U B U^{T} \text {. }
$$

Notice that $U U^{T}=I$. Denote $U^{T} U$ by $\Delta$. It is a semi-infinite diagonal matrix such that its $i$ 'th diagonal element $\delta_{i}$ is 1 if $i$ is in the image of $\phi$, and 0 otherwise.

Lemma 8. $U(H \Delta-\Delta H)=0$ and $U(B \Delta-\Delta B)=0$.

Proof. $(H \Delta-\Delta H)_{i, j}=H_{i, j}\left(\delta_{j}-\delta_{i}\right)$. Assume that $H_{i, j}$ is non-zero. This implies that $j \subset i$. If in addition $i$ is in the image of $\phi$, then its subset $j$ is also in the image of $\phi$, since $\phi$ is an ideal embedding. Hence both $\delta_{i}$ and $\delta_{j}$ are equal to 1 . In other words, $i \in \operatorname{Im}(\phi) \Rightarrow(H \Delta-\Delta H)_{i, j}=0$ for all $j \in \mathcal{Q}$. This implies that $U(H \Delta-\Delta H)=0$. The proof that $U(B \Delta-\Delta B)$ vanishes is similar.

Since $\phi$ is an ideal embedding, we have that $G=U H U^{T}$. According to Lemma 8, $G^{k}=U H^{k} U^{T}$, for all $k \in \mathbb{N}$. But we know that $B=\sum_{k \in \mathbb{N}} \frac{1}{k !} H^{k}$, hence

$$
A=U B U^{T}=\sum_{k \in \mathbb{N}} \frac{1}{k !} U H^{k} U^{T}=e^{G} .
$$

This completes the alternate proof of Lemma 7.

Inverses. We finish by observing that the inverses of $B$ and $A$, i.e., the Möbius matrices of the corresponding posets, are also quite remarkable: they both have entries in $\{-1,0,1\}$. More precisely, we will show that these Möbius matrices have, up to sign, the same entries as their inverses.

Recall that $D$ and $C$ are the diagonal matrices whose non-zero entries are, respectively, $(-1)^{t(i)}$ and $(-1)^{z(i)}, i \in \mathbb{N}$.

Theorem 4. $B^{-1}=D B D$ and $A^{-1}=C A C$.

Proof. Denote the matrix $\left(\begin{array}{cc}1 & 0 \\ 0 & -1\end{array}\right)$ by $D(1)$. Since $(B(1) D(1))^{2}=I(1)$, we have that $I(k)=\left((B(1) D(1))^{2}\right)^{\otimes k}=\left(B(1)^{\otimes k} D(1)^{\otimes k}\right)^{2}$, for all $k \in \mathbb{N}$. This proves that, for all $k \in \mathbb{N}, B(k)^{-1}=D(1)^{\otimes k} B(k) D(1)^{\otimes k}$, and the expression of $B^{-1}$ follows.

Now recall that $A=U B U^{T}$ and observe that $C=U D U^{T}$. This implies that

$$
A C=U B U^{T} U D U^{T}=U B \Delta D U^{T} .
$$

Using the fact that $\Delta$ and $D$ commute, and that $\Delta U^{T}=U^{T}$, we get that $A C=$ $U B D U^{T}$. Hence $(A C)^{2}=U B D \Delta B D U^{T}=U B \Delta D B D U^{T}$. By Lemma 8 we know that $U B \Delta=U \Delta B=U B$. In addition, we already know that $D B D=B^{-1}$. Hence $(A C)^{2}=U B B^{-1} U^{T}=I$. 
Acknowledgements. This work has benefited from the remarks of Roland Bacher. Thanks to the very helpful observations of the referee, an awful lot of awkwardness was, I hope, corrected.

\section{References}

[AS1] J. P. Allouche, and J. Shallit, The Ubiquitous Prouhet-Thue-Morse Sequence, Sequences and their applications (Singapore, 1998), 1-16, Ser. Discrete Math. Theor. Comput. Sci., Springer, London, 1999.

[AS2] J. P. Allouche, and J. Shallit, Automatic Sequences: Theory, Applications, Generalizations, Cambridge University Press, 2003.

[Bó] M. Bóna, A walk through combinatorics. An introduction to enumeration and graph theory, World Scientific Publishing Co., 2002.

[BC] R. Bacher, R. Chapman, Symmetric Pascal matrices modulo p, European J. Combin. 25 (2004), 459-473.

[GKP] R. L. Graham, D. E. Knuth, O. Patashnik, Concrete mathematics, second edition, Addison-Wesley, 1994.

[HJ] R. A. Horn, C. R. Johnson, Topics in matrix analysis, Cambridge university press, 1991.

[Ma] B. B. Mandelbrot, The fractal geometry of nature, W. H. Freeman and Co., 1977.

[Sh] J. Shallit, A generalization of automatic sequences, Theoret. Comput.Sci. 61 (1988), $1-16$.

[Si] W. Sierpinski, Sur une courbe dont tout point est un point de ramification, $C$. $R$. Acad. Sci. Paris 160 (1915), 302-305.

[Sy] J. J. Sylvester, Thoughts on Orthogonal Matrices, Simultaneous Sign-Successions, and Tessellated Pavements in Two or More Colours, with Applications to Newton's Rule, Ornamental Tile-Work, and the Theory of Numbers. Phil. Mag. 34 (1867), $461-475$.

[Ze] E. Zeckendorf, Représentation des nombres naturels par une somme de nombres de Fibonacci ou de nombres de Lucas, Bull. Soc. Roy. Sci. Liège 41 (1972), 179-182. 